\title{
Silencing of $E R K 2$ with Small Interference RNA Regulates the Expression of CXCL1 in Th17 Cell
}

\author{
Wei Wu${ }^{1 *}$, Qian Zhang1, Kaixia Cai'2, Dan Wang², Hui Chen ${ }^{2}$ \\ ${ }^{1}$ Department of Respiratory and Critical Care Medicine, The Second Affiliated Hospital of Xi'an Medical University, Xi'an, China \\ ${ }^{2}$ Department of Respiratory and Critical Care Medicine, The First Affiliated Hospital of Xi'an Medical University, Xi'an, China \\ Email: *wwatp@163.com
}

How to cite this paper: $\mathrm{Wu}, \mathrm{W}$., Zhang, Q., Cai, K.X., Wang, D. and Chen, H. (2021) Silencing of ERK2 with Small Interference RNA Regulates the Expression of CXCL1 in Th17 Cell. Journal of Biosciences and Medicines, 9, 184-194.

https://doi.org/10.4236/jbm.2021.97017

Received: June 20, 2021

Accepted: July 19, 2021

Published: July 22, 2021

Copyright $\odot 2021$ by author(s) and Scientific Research Publishing Inc. This work is licensed under the Creative Commons Attribution International License (CC BY 4.0).

http://creativecommons.org/licenses/by/4.0/

\begin{abstract}
Patients with steroid-resistant asthma had their monocyte-derived TH17 cells collected. The expression levels of ERK2 in the TH17 were silenced and inhibited using ERK2 specific small interfering RNA (siRNA). By screening of CXCL1 and IL-17A in the TH17 culture supernatant, the expression levels of ERK2 and CXCL1 were determined. Using targeted siRNA to inhibit ERK2, the expression of ERK2 in the TH17 was reduced. Furthermore, inhibiting ERK2 hindered CXCL1 expression and decreased CXCL1 and IL-17A production. These findings suggest that ERK2 is involved in the synthesis of CXCL1 and IL-17A, two proteins that play a key role in the pathogenesis of hormone-resistant asthma.
\end{abstract}

\section{Keywords}

Asthma, Th17 Cells and ERK2

\section{Introduction}

Asthma is a common chronic respiratory disease, and its global incidence is increasing year by year. The current treatment strategies include inhaled corticosteroids and $\beta 2$ receptor agonists. The wide application of these treatments has effectively controlled the clinical symptoms of asthma patients and improved their quality of life. However, the mortality rate of asthma patients has not significantly decreased, indicating that there are still many unknown mechanisms for the pathogenesis and treatment of asthma [1] [2].

The current basic and clinical studies have found that the inflammatory cells of asthma airway are mainly eosinophilic infiltration, which respond well to 
hormone therapy. Inhaled or systemic glucocorticoids were also found to be effective and help control asthma symptoms. However, symptoms persist in about $5 \%-10 \%$ of asthma patients who have received adequate glucocorticoid therapy. These patients remain in a poorly controlled state and are affected by hormoneinsensitive or hormone-resistant asthma, subjecting the patient to greater physiological and psychological impact [3]. Airway infiltration with neutrophils as the primary inflammatory cells is the main pathophysiological basis of hormone-resistant asthma. This phenomenon is closely related to a new class of helper Tlymphocytes, namely, Th17 cells. Chemokine (CXC motif) ligand 1 (CXCL1) acts as a key chemoattractant for neutrophils in the pathogenesis of autoimmune diseases [4]. ERK2 is closely related to the occurrence of neutrophil inflammation [5]. However, the specific molecular machinery is not fully understood [6] [7]. Given this, this study aimed to explore the molecular mechanisms of adhesion molecule CXCL1 and the Raf-ERK signaling pathways in Th17 cell-mediated neutrophil airway infiltration, thereby deepening the understanding of hormone-resistant asthma.

\section{Materials and Methods}

\subsection{Patients}

10 patients from the Department of Respiratory and Critical Care Medicine of the First Affiliated Hospital of Xi'an Medical University between January 2017 and March 2018 who were diagnosed with steroid-resistant asthma were included in this investigation [8]. All procedures were performed in accordance with the Declaration of Helsinki for medical research involving human subjects, and written consent from the subjects was obtained. The ethics committee of the First Affiliated Hospital of Xi'an Medical University also approved the study procedures.

\subsection{Isolation and Culture of TH17}

Human peripheral blood mononuclear cells (PBMCs) were isolated from peripheral blood using Ficoll-Paque, as described previously [9]. Furthermore, the PBMCs were isolated from $5 \mathrm{~mL}$ of venous blood using Ficoll-Paque PLUS centrifuge, as described previously [10]. After centrifugation, the PBMCs were collected from the interphase layer and washed four times with RPMI-1640 Medium. Then, the PBMCs were gently re-suspended in $10 \mathrm{~mL}$ of RMPI-1640 Medium supplemented with $10 \%$ FCS and $10-\mathrm{mM}$ EDTA. The PBMCs $\left(1 \times 10^{7}\right.$ cells/mL) suspended in RPMI-1640 Medium supplemented with 10\% (v/v) FBS were incubated at $37^{\circ} \mathrm{C}$ at $5 \% \mathrm{CO}_{2}$ in air with high humidity.

The Th17 cells were induced by PBMC. Briefly, the PBMCs were cultured for 6 days with anti-CD3 (BioLegend Cat\# 300314) plus anti-CD28 mAbs (BD, Cat\#555729, at $5 \mu \mathrm{g} / \mathrm{mL}$ and $2 \mu \mathrm{g} / \mathrm{mL}$, respectively) in the presence of the combination of cytokines and blocking antibodies appropriate for polarization: rhIL-6 (10 ng/mL), rhIL-23 (20 ng/mL), rhTGF- $\beta 1(2 \mathrm{ng} / \mathrm{mL})$, anti-IFN- $\gamma(10 \mu \mathrm{g} / \mathrm{mL})$, 
and anti-IL-4 $(10 \mu \mathrm{g} / \mathrm{mL})$.

\subsection{ERK2-Specific Silencing in Vitro}

On day 7, the TH17 cells were harvested via flow cytometry (BD, Cat\#560436) and suspended in 200- $\mu \mathrm{L}$ serum-free RPMI-1640 Medium and aliquoted into a 24-well plate (Invitrogen Life Technologies). A total of $1 \mu \mathrm{g}$ of each ERK2 small interfering RNA (Qiagen, Hilden, Germany; sense5'-CAGUAGGCUGUAUUCUGGCAGAGAU-3' and antisense 5'-GUTTACGUGACACGUUCGGAGAATT-3') and negative control (scrambled) siRNA (sense5'-UAGGCGCAGCUCCGGAUCGDTT-3' and antisense 5 '-CGAUCCGGAGCUGCGCCUADTT-3') were incubated separately, with $5-\mu \mathrm{L}$ Lipofectamine (Genlantis, Inc., San Diego, CA, USA) in 100- $\mu \mathrm{L}$ serum-free RPMI1640 Medium at room temperature for $5 \mathrm{~min}$. The ERK2-specific and control siRNA mixtures were then added to the respective 200- $\mu \mathrm{L}$ DC cell cultures. After a $4 \mathrm{~h}$ incubation at $37^{\circ} \mathrm{C}$, an equal volume of $200-\mu \mathrm{L}$ RPMI-1640 Medium supplemented with $20 \%$ FCS was added to the cells. siRNA transfection and silencing of the expression of ERK2 in the TH17 cells were performed according to the methods described in a previous study [11].

\subsection{RT-qPCR}

Total RNA was isolated from the TH17 cells, which were collected $72 \mathrm{~h}$ after the siRNA-ERK2 transfection, using RNeasy Mini Kits (Qiagen, Valencia, CA, USA) containing TRIzol reagent for extraction (Gibco Life Technologies, Carlsbad, CA, USA). The total RNA was then reverse-transcribed into cDNA using a reverse transcription kit at $50^{\circ} \mathrm{C}$ for $30 \mathrm{~min}$. The cDNA was amplified using Promega PCR Single-Step Kit (Promega Corporation, Madison, WI, USA) according to the manufacturer's protocols. The following primers (Invitrogen Life Technologies) were used: ERK2, sense 5'-CTAACGTTCTGCACCG TGAC-3' and antisense 5'-GATCACAAGTGGTGTTCAGCAG-3'; $\beta$-actin, sense 5'-CCTCATGAAGATCCTGACCG-3', and antisense 5'-ACCGCTCATTGCCGATAGTG-3'. $\beta$-actin served as the internal control. Quantification of the PCR products was performed using FTC-2000 (Shanghai Funglyn Biotech Co., Ltd., Shanghai, China). The threshold cycle (Ct) of each sample was recorded as a quantitative measure of the amount of PCR product in the sample. Relative changes in the expression levels were calculated using the 2- $\Delta \Delta \mathrm{CT}$ method [12].

\subsection{Western Blot}

The TH17 cells were collected $72 \mathrm{~h}$ after the siRNA-ERK2 transfection. Total protein was extracted using ice-cold radioimmunoprecipitation lysis buffer (Santa Cruz, Biotechnology, Inc., Dallas, TX, USA). The protein concentrations were quantified using the Bradford method (Quick Start ${ }^{\mathrm{Tw}}$; Bio-Rad, Hercules, CA, USA). Cytoplasmic protein samples (20 $\mu \mathrm{g})$ were separated via 15\% SDS-PAGE and 
semi-dry-transferred onto polyvinylidene difluoride membranes (EMD Millipore, Billerica, MA, USA). The membranes were blocked with $5 \%$ bovine serum albumin (Santa Cruz, Biotechnology, Inc.) for $1 \mathrm{~h}$ at room temperature. The blots were then incubated at $4^{\circ} \mathrm{C}$ overnight with the following primary antibodies: anti-ERK2 (ab231085) and anti-GAPDH (ab8245 Abcam Biotechnology, Inc.) at a 1:1000 dilution in Tris-buffered saline with Tween-20 (TBST). GAPDH was used as a loading control. The blots were washed with TBST and then incubated with an anti-rabbit immunoglobulin G-horseradish peroxidase-conjugated secondary antibody (1:20,000 dilution in TBST; Pierce Biotechnology, Inc., Rockford, IL, USA) for $1 \mathrm{~h}$ at room temperature. After further washing with TBST, the blots were visualized using enhanced chemiluminescence (GE Healthcare Life Sciences, Little Chalfont, UK) and densitometrical quantified using a western blotting detection system (GE Healthcare Life Sciences). All results were normalized to GAPDH. Heat-induced epitope retrieval was conducted.

\subsection{ELISA}

The levels of inflammatory cytokines in the Th17 cell culture supernatants were analyzed via ELISA using ELISA kits for IL-17a and CXCL-1 (R\&D Systems, Inc.) according to the manufacturer's protocols. A total of five mice were used for each group per experiment.

\subsection{Statistical Analysis}

Statistical analysis was conducted using the SPSS software version 20.0 (SPSS Inc., Chicago, IL, USA). Data were analyzed using T-Test, followed by LSD significant difference test. A value of $\mathrm{P}<0.05$ (two-tailed test) was considered statistically significant. The results are expressed as mean \pm standard deviation unless otherwise stated.

\section{Results}

\subsection{ERK2-Specific siRNA Effectively Inhibits the Gene Expression of ERK2}

To investigate whether ERK2-specific siRNA silenced the expression of ERK2 in the present study, the mRNA expression levels of ERK2 were detected via RT-qPCR. The mRNA expression levels of ERK2 were downregulated in the ERK2-specific siRNA group $72 \mathrm{~h}$ after transfection compared with the control group (Figure 1(a) and Figure 1(b)). This indicated that ERK2-specific siRNA effectively inhibited the gene expression of ERK2.

\subsection{ERK2-Specific siRNA Effectively Inhibited the Protein Expression Levels of ERK2}

Western blot analysis revealed that the protein expression of ERK2 in the ERK2-siRNA group downregulated compared with the control and scrambledsiRNA group (Figure 2(a) and Figure 2(b)). These results indicated a signifi- 
cant reduction in the expression of ERK2 in the ERK2-siRNA group.

\subsection{ERK2-siRNA Reduces the Gene Expression of CXCL1}

To investigate whether ERK2-specific siRNA affected the gene expression levels of CXCL1, the mRNA levels of CXCL1 were detected via RT-qPCR. The mRNA expression levels of CXCL1 were downregulated in the ERK2-siRNA group $72 \mathrm{~h}$ after transfection compared with the control group (Figure 3 ).

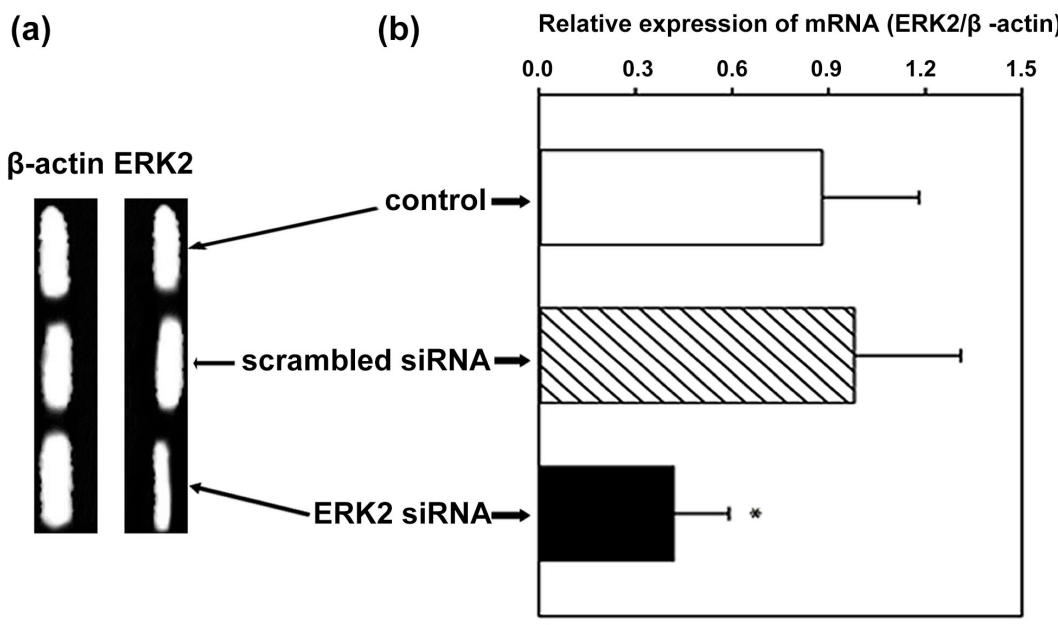

Figure 1. The mRNA levels of ERK2 inTH17 cells. (a) mRNA expression levels of ERK2 were downregulated $72 \mathrm{~h}$ post-transfection in the ERK2-siRNA group. (b) Data are presented as the mean \pm SD of the relative intensity of ERK2 normalized against $\beta$-actin. ${ }^{\star} \mathrm{P}<0.05$, vs. control and scrambled-siRNA groups. The experiment was performed three times and representative results are presented. ERK2, extracellular regulated protein kinases 2; TH17, T helper 17 cell; siRNA, small interfering RNA; SD, standard deviation.

(a)

(b) ERK2 protein levels (relative amount)

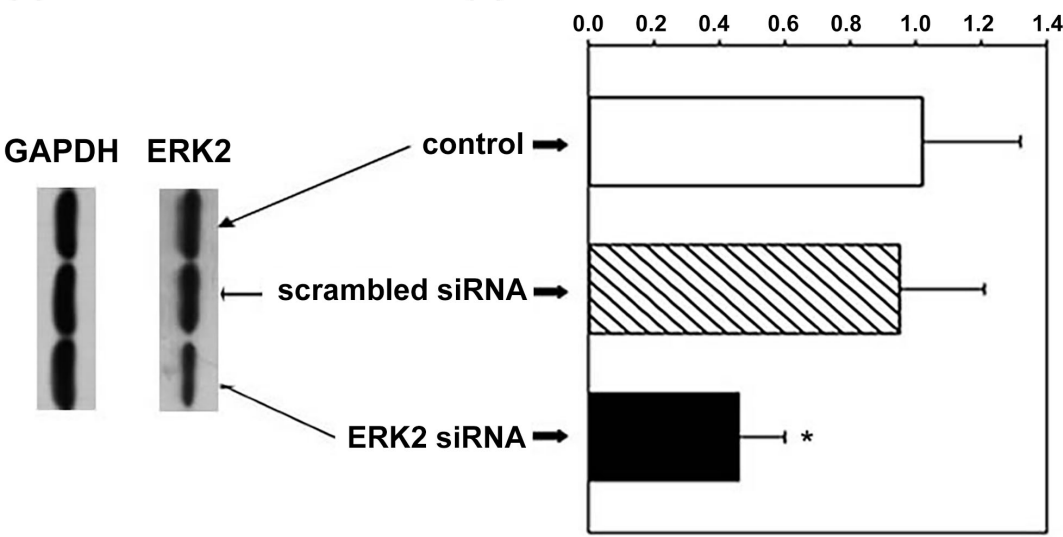

Figure 2. Analysis of protein expression levels of ERK2 in TH17 cells. (a) Protein expression levels of ERK2 in TH17 cells $72 \mathrm{~h}$ post-transfection in the ERK2-siRNA group. (b) Data are presented as the mean \pm SD of the relative intensity of ERK2 normalized against GAPDH. ${ }^{\star} \mathrm{P}<0.05$, vs. control and scrambled-siRNA groups. The experiment was performed three times and representative results are presented. 
(a)

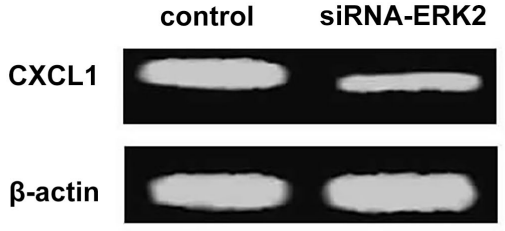

(b)

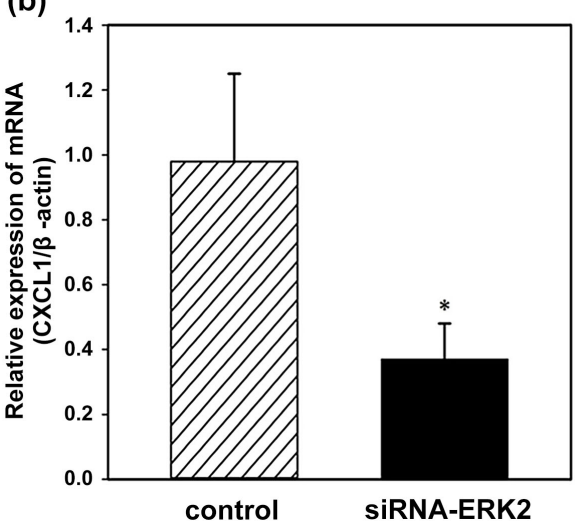

Figure 3. The mRNA levels of CXCL1 in TH17 cells. (a) mRNA expression of c-kit was downregulated $72 \mathrm{~h}$ post-transfection with ERK2 siRNA. (b) Data are presented as the mean \pm SD of the relative intensity of CXCL1 normalized against $\beta$-actin. TH17 cells were collected $72 \mathrm{~h}$ post-transfection with ERK2 siRNA or phosphate-buffered saline vehicle. ${ }^{\star} \mathrm{P}<0.05$, vs. control group. The experiment was performed three times and representa-tive results are presented. ERK2, extracellular regulated protein kinases 2; TH17, T helper 17 cell; siRNA, small interfering RNA; SD, standard deviation.

\subsection{ERK2-siRNA Reduces the Protein Expression of CXCL1}

The protein levels of CXCL1 were detected via western blot analysis and were downregulated in the ERK2-siRNA group compared with the control group. A significant difference was observed in the expression levels of CXCL1 between the ERK2-siRNA group and the control group (Figure 4).

\subsection{ERK2-siRNA Regulates the Production of Cytokines}

The concentration of IL-17A and CXCL1 in the culture supernatants of the TH17 cells significantly decreased in the ERK2-siRNA group compared with the control and scrambled-siRNA group (Figure 5).

\section{Discussion}

Since hormone-resistant asthma poses a significant challenge to clinical treatment. It is important to elucidate the pathogenesis of hormone-resistant asthma. In the present study, specific siRNA was used to silence the expression of ERK2. ERK2 silencing was found to downregulate the expression of CXCL1. This suggests that ERK2 is involved in the regulation of CXCL1 expression, which has 
(a)

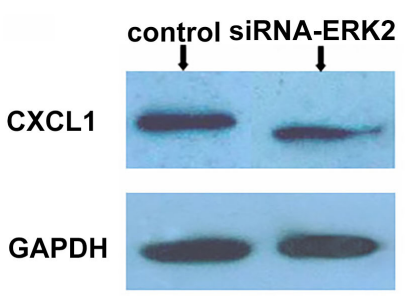

(b)

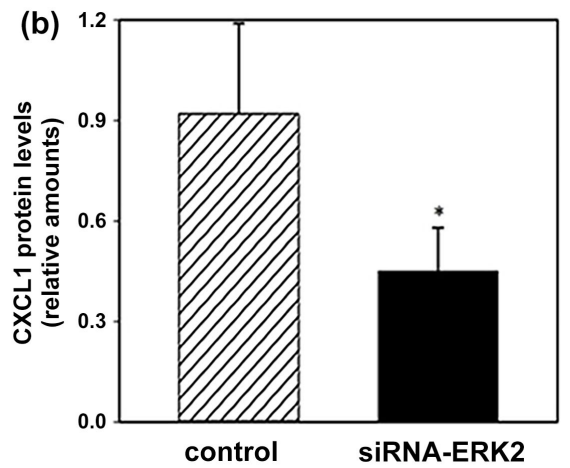

Figure 4. The protein expression levels of CXCL1 in TH17 cells. (a) Protein expression of CXCL1 in TH1772 h post-transfection. (b) Data are presented as the mean \pm SD of the relative intensity of CXCL1 normalized against GAPDH. TH17 were collected $72 \mathrm{~h}$ post-transfection with ERK2 siRNA or phosphate-buffered saline vehicle. ${ }^{\star} \mathrm{P}<0.05$, vs. control group. The experiment was performed three times and representa-tive results are presented.
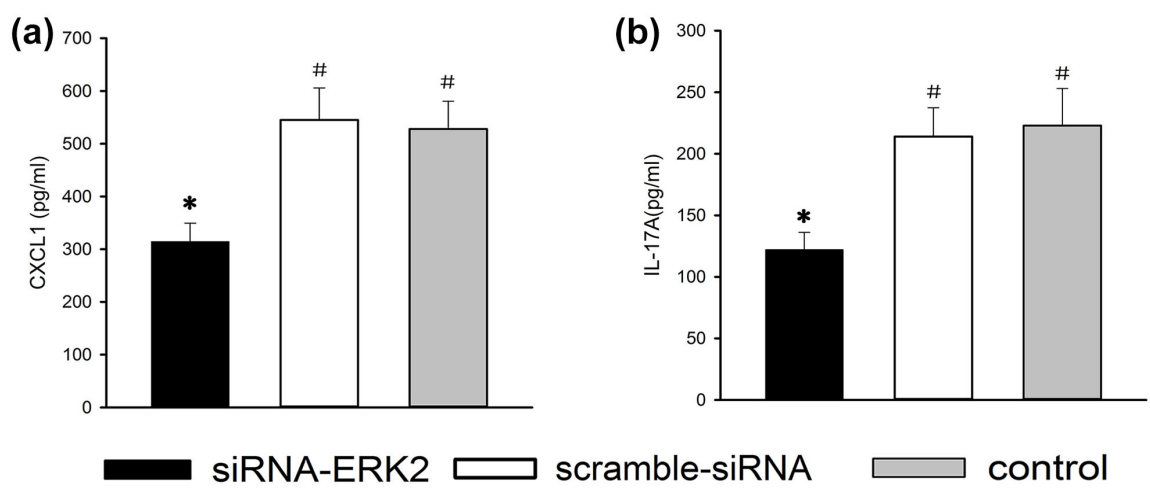

Figure 5. ERK2 siRNA selectively regulates cytokine production in TH17 cells. The levels of (a) CXCL1 and (b) IL-17A in the supernatants of the TH17 were measured using ELISA analysis. The supernatants were obtained $72 \mathrm{~h}$ post-transfection with the ERK2 siRNA or a phosphate-buffered saline vehicle. ${ }^{\star} \mathrm{P}<0.05$, vs. control; ${ }^{\#} \mathrm{P}>0.05$, siRNA group vs. scramble-siRNA group (Student's t-test). Data are presented as the mean \pm standard error of the mean of three independent experiments. TH17, T helper 17 cell; siRNA, small interfering RNA; SD, standard deviation.

not been observed before. Numerous studies have been conducted to investigate the role of ERK2 during the development of asthma and have indicated that ERK2 is closely associated with the neutrophilic infiltration of the airways and the proliferation of airway smooth muscle cells [13] [14]. In addition, ERK2 mediates the production of CXCL8, indicating that ERK2 is closely related to the transcriptional regulation of the CXC family [15]. ERK2 can promote the expressions of CXCL1 and CXCL8, and then CXCL1 and CXCL8 can induce neutrophils to recruit airways. CXC chemokine(s) have been demonstrated to play an important role in recruiting neutrophils to inflamed tissues [16].

When the expression of ERK2 is inhibited by specific siRNA, which restrains the production of IL-17A, IL-17A can stimulate airway structural cells, including bronchial epithelial cells, to secrete CXCL8 and CXCL1 [17]. In the present in- 
vestigation, we employed specific siRNA silencing of ERK2, which downregulated the expression of CXCL1. In other words, ERK2 is closely involved in the expression and regulation of CXCL1. IL-17 is also another chemokine that attracts neutrophils. The IL-17 levels are elevated in the subjects with hormoneresistant asthma compared with those with controlled asthma or normal controls. Recent reports suggest that uncontrolled asthma is associated with elevated numbers of neutrophils and levels of neutrophil-attracting chemokines IL-8 and IL-17 in BAL fluids [18]. Subjects with asthma have greater percentages of IL-17A-positive PBMC compared with healthy control subjects [19] [20]. The number of bronchial IL-17F-positive cells correlates with the number of bronchial neutrophils and FEV1 (\% predicted) [21]. Therefore, IL-17A and IL-17F are the central marker cytokines of TH17 cells, suggesting that the TH17 cells are closely related to hormone-resistant asthma.

Silencing the ERK2 expression via specific siRNA, and we found the production of IL-17A was correspondingly observed to decrease. IL-17 can promote the accumulation of neutrophils in the airways via Granulocyte-Macrophage Colony-Stimulating Factor (GM-CSF) [22]. GM-CSF has the effect of prolonging the survival of neutrophils by inhibiting their apoptosis [23]. The IL-17-induced GM-CSF release is probably due to the inhibition of the transcription factor Nuclear Factor (NF)- $\kappa B$ [24]. The activation of NF- $\kappa B$ in the airway epithelial cells can produce chemokines that play an important role in neutrophil recruitment and inflammation in neutrophilic asthma [25]. MEK5DD and MEK1R4F synergize to activate an NF- $\mathrm{KB}$-sensitive reporter. MEK5 and ERK5 are involved in the RafBXB activation of NF- $\kappa B$. Moreover, ERK5 and ERK2 cooperate to re-

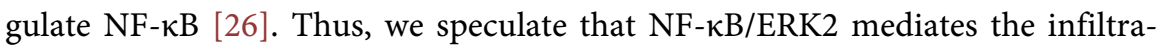
tion of neutrophils in the airway induced by TH17 cells. Another study found that IL-17 and TNF- $\alpha$ promote the accumulation of neutrophils in the airways and have implications for human asthma and COPD [27]. ERK2, rather than ERK1, mediates LPS-induced GM-CSF expression in macrophages [28]. The $\mathrm{NF}-\mathrm{\kappa B} / \mathrm{ERK} 2$ signaling pathway is involved in the regulation of neutrophil recruitment mediated by CXCL1, TNF- $\alpha$, and GM-CSF, which together form a complex regulatory network.

In conclusion, this investigation demonstrated that ERK2 is important in the chemotaxis of neutrophils mediated by the TH17 cells. Inhibiting the expression of ERK2 with specific siRNA resulted in the downregulation of CXCL1 and reduced expression of IL-17A. These effects were associated with hormone-insensitive asthma mediated by neutrophils. However, CXCL1, TNF- $\alpha$, and GM-CSF interact to induce neutrophil-based asthma, which still needs further investigation.

\section{Acknowledgements}

This study was supported by grants 16JK1646 and 15JK1618 from Special Scientific Research Project of Education Department of Shaanxi Provincial Department. 


\section{Conflicts of Interest}

The authors declare no conflicts of interest regarding the publication of this paper.

\section{References}

[1] Praveen, M. and Mark, D.S. (2010) Analytic Review: Management of Life-Threatening Asthma in Adults. Journal of Intensive Care Medicine, 25, 3-15. https://doi.org/10.1177/0885066609350866

[2] Vanessa, L.H. and Pamela, R.W. (2009) Asthma Epidemiology, Pathophysiology, and Initial Evaluation. Pediatrics in Review, 30, 331-336.

https://doi.org/10.1542/pir.30-9-331

[3] Szefler, S.J. and Leung, D.Y. (1997) Glucocorticoid-Resistant Asthma: Pathogenesis and Clinical Implications for Management. European Respiratory Journal, 10, 1640-1647. https://doi.org/10.1183/09031936.97.10071640

[4] Sawant, K.V., Poluri, K.M., Dutta, A.K., Sepuru, K.M., Troshkina, A., Garofalo, R.P., et al. (2016) Chemokine CXCL1 Mediated Neutrophil Recruitment: Role of Glycosaminoglycan Interactions. Scientific Reports, 6, Article No. 33123.

https://doi.org/10.1038/srep33123

[5] Senger, K., Pham, V.C., Varfolomeev, E., Hackney, J.A., Corzo, C.A., Collier, J., et al. (2017) The Kinase TPL2 Activates ERK and p38 Signaling to Promote Neutrophilic Inflammation. Science Signal, 10, Article No. eaah4273.

https://doi.org/10.1126/scisignal.aah4273

[6] Zhang, Y., Zhang, L., Wu, J., Di, C. and Xia, Z. (2013) Heme Oxygenase-1 Exerts a Protective Role in Ovalbumin-Induced Neutrophilic Airway Inflammation by Inhibiting Th17 Cell-Mediated Immune Response. Journal of Biological Chemistry, 288, 34612-34626. https://doi.org/10.1074/jbc.M113.494369

[7] Besnard, A.-G., Togbe, D., Couillin, I., Tan, Z., Zheng, S.G., Erard, F., et al. (2012) Inflammasome-IL-1-Th17 Response in Allergic Lung Inflammation. Journal of Molecular Cell Biology, 4, 3-10. https://doi.org/10.1093/jmcb/mjr042

[8] Corrigan, C.J. and Loke, T.-K. (2007) Clinical and Molecular Aspects of Glucocorticoid Resistant Asthma. Therapeutics and Clinical Risk Management, 3, 771-787.

[9] Pham, T.N.Q., King, D., Macparland, S.A., McGrath, J.S., Reddy, S.B., Bursey, F.R., et al. (2008) Hepatitis C Virus Replicates in the Same Immune Cell Subsets in Chronic Hepatitis C and Occult Infection. Gastroenterology, 134, 812-822. https://doi.org/10.1053/j.gastro.2007.12.011

[10] Corkum, C.P., Ings, D.P., Burgess, C., Karwowska, S., Kroll, W. and Michalak, T.I. (2015) Immune Cell Subsets and Their Gene Expression Profiles from Human PBMC Isolated by Vacutainer Cell PreparationTube $\left(\mathrm{CPT}^{\mathrm{TM}}\right)$ and Standard Density Gradient. BMC Immunology, 16, Article No. 48. https://doi.org/10.1186/s12865-015-0113-0

[11] Zheng, X., Vladau, C., Zhang, X., Suzuki, M., Ichim, T.E., Zhang, Z.X., Li, M., Carrier, E., Garcia, B., Jevnikar, A.M. and Min, W.P. (2009) A Novel in Vivo siRNA Delivery System Specififically Targeting Dendritic Cells and Silencing CD40 Genes for Immunomodulation. Blood, 113, 2646-2654. https://doi.org/10.1182/blood-2008-04-151191

[12] Livak, K.J. and Schmittgen, T.D. (2001) Analysis of Relative Gene Expression Data Using Real-Time Quantitative PCR and the $2^{-\Delta \Delta \mathrm{C}_{\mathrm{T}}}$ Method. Methods, 25, 402-408. https://doi.org/10.1006/meth.2001.1262 
[13] Whelchel, A., Evans, J. and Posada, J. (1997) Inhibition of ERK Activation Attenuates Endothelin-Stimulated Airway Smooth Muscle cell Proliferation. American Journal of Respiratory Cell and Molecular Biology, 16, 589-596. https://doi.org/10.1165/ajrcmb.16.5.9160841

[14] Robins, S., Roussel, L., Schachter, A., Risse, P.A., Mogas, A.K., Olivenstein, R., Martin, J.G., Hamid, Q. and Rousseau, S. (2011) Steroid-Insensitive ERK1/2 Activity drives CXCL8 Synthesis and Neutrophilia by Airway Smooth Muscle. American Journal of Respiratory Cell and Molecular Biology, 45, 984-990. https://doi.org/10.1165/rcmb.2010-0450OC

[15] Jo, E.J., Lee, H.-Y., Lee, Y.-N., Kim, J.I., Kang, H.-K., Park, D.-W., Baek, S.-H., Kwak, J.-Y. and Bae, Y.-S. (2004) Group IB Secretory Phospholipase A2 Stimulates CXC Chemokine Ligand 8 Production via ERK and NF-Kappa B in Human Neutrophils. The Journal of Immunology, 173, 6433-6439.

https://doi.org/10.4049/jimmunol.173.10.6433

[16] Shanley, T.P., Schmal, H., Warner, R.L., Schmid, E., Freidl, H.P. and Ward, P.A. (1997) Requirement for C-X-C Chemokines (Macrophage Inflammatory Protein-2 and Cytokine-Induced Neutrophil Chemoattractant) in IgG Immune Complex- Induced Lung Injury. The Journal of Immunology, 158, 3439-3448.

[17] Halwani, R., Al-Muhsen, S. and Hamid, Q. (2013) T Helper 17 Cells in Airway Diseases from Laboratory Bench to Bedside. Chest, 143, 494-501.

https://doi.org/10.1378/chest.12-0598

[18] Hosoki, K., Boldogh, I. and Sur, S. (2016) Neutrophil Recruitment by Allergens Contribute to Allergic Sensitization and Allergic Inflammation. Current Opinion in Allergy and Clinical Immunology, 16, 45-50. https://doi.org/10.1097/ACI.0000000000000231

[19] Chakir, J., Shannon, J., Molet, S., Fukakusa, M., Elias, J., Laviolette, M., Boulet, L.P. and Hamid, Q. (2003) Airway Remodeling-Associated Mediators in Moderate to Severe Asthma: Effect of Steroids on TGF-Beta, IL-11, IL-17, and Type I and Type III Collagen Expression. Journal of Allergy and Clinical Immunology, 111, 1293-1298. https://doi.org/10.1067/mai.2003.1557

[20] Nanzer, A.M., Chambers, E.S., Ryanna, K., Richards, D.F., Black, C., Timms, P.M., Martineau, A.R., Griffiths, C.J., Corrigan, C.J. and Hawrylowicz, C.M. (2013) Enhanced Production of IL-17A in Patients with Severe Asthma Is Inhibited by 1a,25-Dihydroxyvitamin D3 in a Glucocorticoid-Independent Fashion. Journal of Allergy and Clinical Immunology, 132, 297-304.E3. https://doi.org/10.1016/j.jaci.2013.03.037

[21] Sorbello, V., Ciprandi, G., Di Stefano, A., Massaglia, G.M., Favata, G., Conticello, S., Malerba, M., Folkerts, G., Profita, M., Rolla, G., et al. (2015) Nasal IL-17F Is Related to Bronchial IL-17F/Neutrophilia and Exacerbations in Stable Atopic Severe Asthma. Allergy, 70, 236-240. https://doi.org/10.1111/all.12547

[22] Laan, M., Prause, O., Miyamoto, M., Sjostrand, M., Hytonen, A.M., Kaneko, T., Lotvall, J. and Linden, A. (2003) A Role of GM-CSF in the Accumulation of Neutrophils in the Airways Caused by IL-17 and TNF-a. European Respiratory Journal, 21, 387-393. https://doi.org/10.1183/09031936.03.00303503

[23] Cox, G., Gauldie, J. and Jordana, M. (1992) Bronchial Epithelial Cell-Derived Cytokines (G-CSF and GM-CSF) Promote the Survival of Peripheral Blood Neutrophils in Vitro. American Journal of Respiratory Cell and Molecular Biology, 7, 507-513. https://doi.org/10.1165/ajrcmb/7.5.507

[24] Brattsand, R. and Linden, M. (1996) Cytokine Modulation by Glucocorticoids: Mechanisms and Actions in Cellular Studies. Alimentary Pharmacology \& Therapeu- 
tics, 10, 81-90. https://doi.org/10.1046/j.1365-2036.1996.22164025.x

[25] Wan, R., Jiang, J., Hu, C., Chen, X., Chen, C., Zhao, B., Hu, X., Zheng, Z. and Li, Y. (2020) Neutrophil Extracellular Traps Amplify Neutrophil Recruitment and Inflammation in Neutrophilic Asthma by Stimulating the Airway Epithelial Cells to Activate the TLR4/ NF-kB Pathway and Secrete Chemokines. Aging, 12, 16820-16836. https://doi.org/10.18632/aging.103479

[26] Pearson, G., English, J.M., White, M.A. and Cobb, M.H. (2001) ERK5 and ERK2 Cooperate to Regulate NF- $\mathrm{KB}$ and Cell Transformation. Journal of Biological Chemistry, 276, 7927-7931. https://doi.org/10.1074/jbc.M009764200

[27] Aaron, S.D., Angel, J.B., Lunau, M., Wright, K., Fex, C., Le Saux, N., et al. (2001) Granulocyte Inflammatory Markers and Airway Infection during Acute Exacerbation of Chronic Obstructive Pulmonary Disease. American Journal of Respiratory and Critical Care Medicine, 163, 349-355.

https://doi.org/10.1164/ajrccm.163.2.2003122

[28] Chang, S.-F., Lin, S.-S., Yang, H.-C., Chou, Y.-Y., Gao, J.-I. and Lu, S.-C. (2015) LPS-Induced G-CSF Expression in Macrophages Is Mediated by ERK2, but Not ERK1. PLOS ONE, 10, e0129685. https://doi.org/10.1371/journal.pone.0129685 